\title{
THE GENUS BAIJIANIA (CUCURBITACEAE)
}

\author{
W.J.J.O. DE WILDE \& B.E.E. DUYFJES \\ Nationaal Herbarium Nederland, Universiteit Leiden branch, \\ P.O. Box 9514, 2300 RA Leiden, The Netherlands; e-mail: dewilde@nhn.leidenuniv.nl
}

SUMMARY

The genus Baijiania, originally thought to be indigenous in China and Borneo, appears to be restricted to Borneo. The only species is Baijiania borneensis, with two varieties, the type variety and var. paludicola Duyfjes, var. nov.

Key words: Baijiania, Cucurbitaceae, Borneo.

\section{INTRODUCTION}

The cucurbitaceous genus Baijiania A.M. Lu \& J.Q. Li (Li, 1993: 50) was described from China and Borneo. A Bornean species, Thladiantha borneensis Merr. was chosen as type, which in habit most closely resembles species from China. However, the flower characters of the type species indicate that the plants from Borneo cannot belong to the same genus as those species from China. For the Chinese species therefore a new genus name will be proposed (in prep.).

Baijiania is distinct from the proposed new Chinese genus in the staminate flower morphology. In particular by the absence of adaxial basal petal scales, the shape of the stamens, and by the presence of a 3-parted basal disc. Baijiania is monotypic, with two varieties, restricted to Sabah and E Kalimantan.

\section{BAIJIANIA}

Baijiania A.M. Lu \& J.Q. Li, in Li (1993) 50, p.p. for the type only; excluding material from China. - Type: Baijiania borneensis (Merr.) A.M. Lu \& J.Q. Li.

Thladiantha Bunge subg. Microlagenaria C. Jeffrey (1962) 363, p.p.

Siraitia Merr. subg. Microlagenaria (C. Jeffrey) A.M. Lu \& Zhi Y. Zhang (1984) 30, p.p., for the specimens from Borneo only.

Plants with tuberous rhizome (?). Hairs not glandular. Tendrils coiling both below and above point of branching. Leaf blade unlobed. Male inflorescences racemose (sometimes \pm branched). Male flowers: receptacle tube about as long as wide or longer than wide; stamens 5, inserted below the throat of the receptacle, two pairs with filaments connate at base, one stamen solitary, thecae somewhat curved, glabrous; filaments short; petals longer than sepals, creamy or greenish white, basal adaxial scales absent; disc at bottom of receptacle conspicuous, 3-parted. Female flowers in a few-flowered 
raceme; staminodes present. Fruit globose, of medium size, c. $4 \mathrm{~cm}$ diam., pulp not sweet tasting. Seeds of medium size, \pm ovoid, \pm compressed, smooth, not margined.

Distribution - Malesia: Borneo; one species with two varieties.

\section{Baijiania borneensis (Merr.) A.M. Lu \& J.Q. Li}

Baijiania borneensis (Merr.) A.M. Lu \& J.Q. Li, in Li (1993) 50, for the type only; Beaman et al. (2001) 206, pl. 15 B , C. - Thladiantha borneensis Merr.(1929) 298. - Siraitia borneensis (Merr.) A.M. Lu \& Zhi Y. Zhang (1984) 31, p.p., specimens from Borneo only; Zhi Y. Zhang \& A.M. Lu (1989) 26, f. 26, 27. - Type: Villamil 308 (UC, not seen; iso BO, K, PNH lost?), Sabah.

Climber, 3-6 m, sparsely grey pubescent and minutely papillose on young parts, hairs c. $1 \mathrm{~mm}$ long; leafy stem 2.5-4 mm diam. Tendrils $10-25 \mathrm{~cm}$ long. Probract absent. Leaves: petiole $3-5 \mathrm{~cm}$ long; blade ovate or ovate-oblong, $10-20(-30)$ by $5-14(-17)$ $\mathrm{cm}$, base cordate, apex acute-acuminate, margin entire or faintly lobulate-dentate near the base; (3-)5-palminerved and 2 or 3 pairs of nerves from midnerve; cystoliths adaxially; greenish on drying. Flowers dioecious. Male inflorescences $8-15 \mathrm{~cm}$ long, peduncle 4-8 cm long, flower-bearing part 4-10 cm long, bracts persistent, elliptic (-oblong) or spathulate, 3-10(-15) mm long, rounded, entire, conspicuously veined; pedicel 5-15(-30) $\mathrm{mm}$ long, faintly articulate $3-10 \mathrm{~mm}$ below perianth, the portion below articulation persistent; open flower 5-8 mm diam.; receptacle cup-shaped, $2.5-4.5$ by $2-4 \mathrm{~mm}$; stamens inserted $0.5(-1) \mathrm{mm}$ below the rim of the receptacle, filaments $0.5-1 \mathrm{~mm}$ long; anthers (thecae) 2-3 mm long, half included or exserted; sepals narrowly triangular-linear, $1.5-3 \mathrm{~mm}$ long, patent or recurved; petals imbricate, elliptic, rounded, 2-5 mm long, 3(-5)-nerved, papillose; disc conspicuous, central, basal, consisting of 3 contiguous subconical parts, 1-1.5 mm high. Female inflorescences few (1-3)-flowered, peduncle 2-8 cm long; bracts like in male. Female flowers like male flowers; ovary ellipsoid, $1.5 \mathrm{~mm}$ long, passing with short neck (c. $1 \mathrm{~mm}$ long) into shallow hypanthium tube c. $1 \mathrm{~mm}$ long; style conspicuous, c. $2 \mathrm{~mm}$ long, stigma with 3 thick notched lobes; staminodes small, 0.5-1 mm long, in 2 pairs and one solitary. Fruit solitary or 2 or 3 together, peduncle $2.5-10 \mathrm{~cm}$ long, bracts persistent; fruiting pedicel $0.5-1.5 \mathrm{~cm}$ long, pubescent; fruit globose, $3-4.5 \mathrm{~cm}$ diam., orange, grey-white soft hairy, hairs 1-2 mm long; pericarp thin, pergamentaceous, endocarp pulpy-juicy. Seeds many, (broadly) ovoid, rounded, truncate, smooth, unmargined, c. $5 \mathrm{~mm}$ in diameter.

Distribution - Borneo: Sabah, SE Kalimantan.

Habitat \& Ecology - Forest margins, roadsides, only in recently cleared or moderately disturbed areas; 0-1000 $\mathrm{m}$ altitude. Flowering and fruiting throughout the year.

\section{KEY TO THE VARIETIES}

1a. Male inflorescences simple, racemose or \pm branched, flowers lax; pedicels 1-3 $\mathrm{cm}$ long. Male perianth $8-10 \mathrm{~mm}$ diam.; receptacle tube about as long as wide, c. $4 \mathrm{~mm}$ long; anthers (thecae) c. $3 \mathrm{~mm}$ long. - Dry land forest a. var. borneensis

b. Male inflorescences a narrow raceme, densely set with flowers; pedicels $0.5-1$ cm long. Male perianth 5-6 mm diam.; receptacle tube longer than wide, c. $3 \mathrm{~mm}$ long; anthers (thecae) 1.5-2 mm long. - Peat swamp forest . b. var. paludicola 


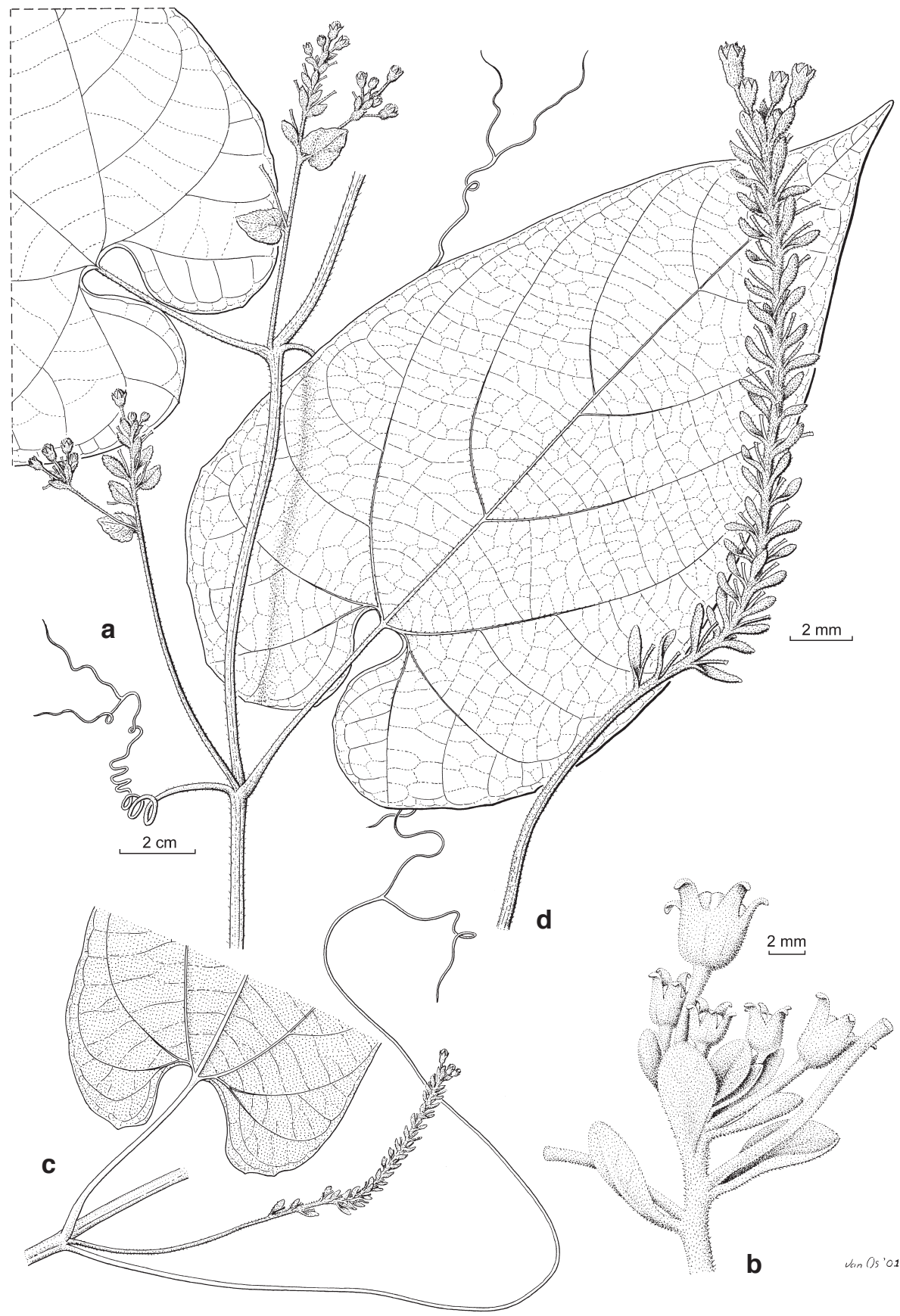

Fig. 1. a, b: Baijiania borneensis A.M. Lu \& J.Q.Li var. borneensis. a. Twig with male inflorescences; b. detail of male inflorescence. - c, d: B. borneensis A.M. Lu \& J.Q. Li var. paludicola Duyfjes. c. Twig with male inflorescence; d. male inflorescence (a, b: SAN (De Wilde \& Duyfjes) 139471; c, d: Kadir A 2520; all drawn by Jan van Os). 

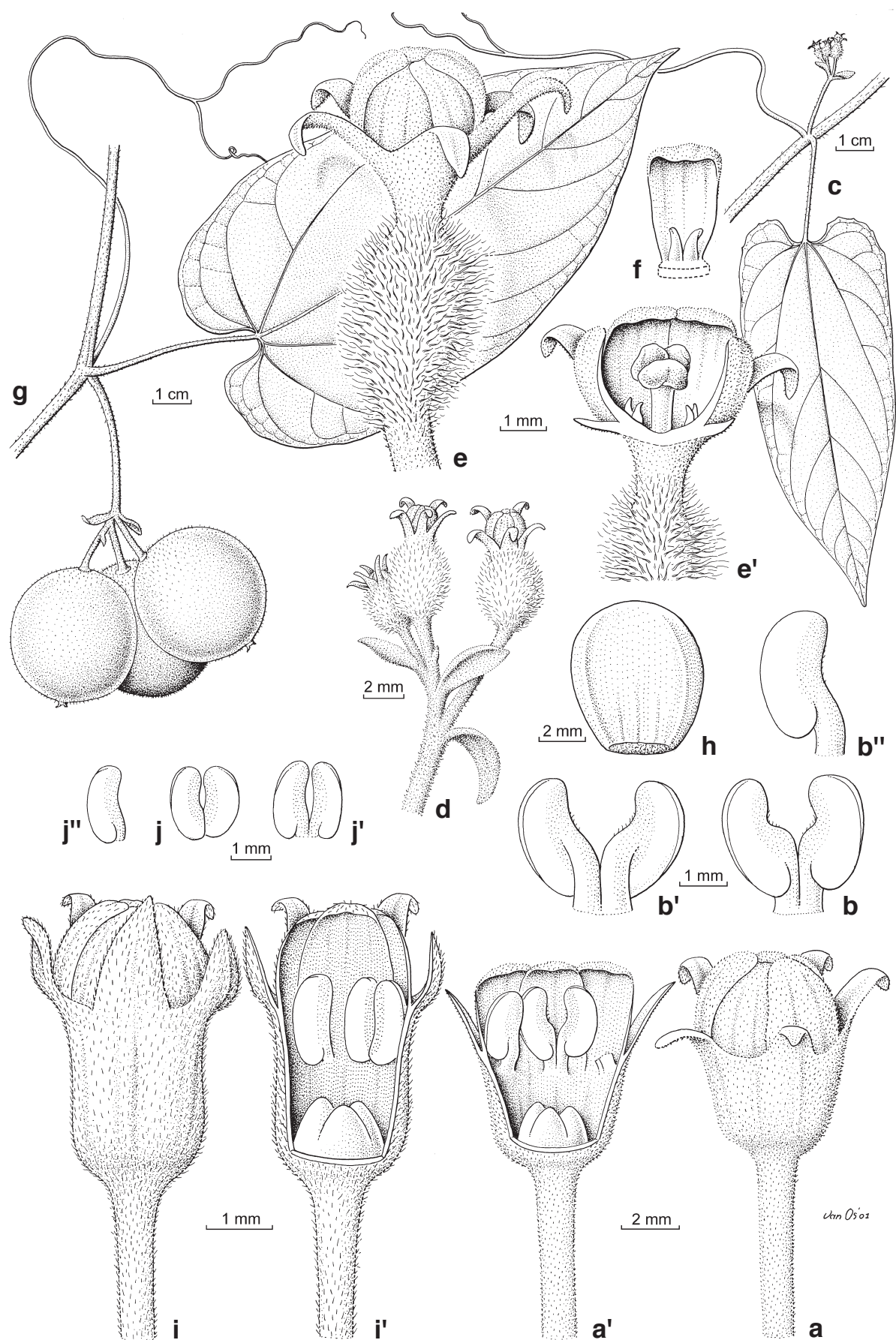
a. var. borneensis - Fig. 1a, b; 2a-h

Male inflorescences grey, yellow or pale brown pubescent, simple or \pm (1- or) 2branched, each branch 10-20-flowered; bracts lanceolate or obovate-spathulate, 7-15 $\mathrm{mm}$ long; pedicels 7-20(-30) $\mathrm{mm}$ long, longer than bracts. Male perianth $8-10 \mathrm{~mm}$ diam., receptacle c. 4 by $4 \mathrm{~mm}$, sepals $2.5-3 \mathrm{~mm}$ long, petals $5-6$ by $3.5 \mathrm{~mm}$; filaments c. $1.5 \mathrm{~mm}$ long, anthers (thecae) c. $3 \mathrm{~mm}$ long.

Distribution - Borneo: Sabah, SE Kalimantan.

Habitat \& Ecology - Forest edges, in scrub in forest gaps, in (open or) shaded places; 90-1000 m altitude. Flowering and fruiting throughout the year.

Note - Female flowers and fruit only known from specimens which belong to var. borneensis.

b. var. paludicola Duyfjes, var. nov. - Fig. 1c, d, 2i-j"

A varietate borneensi in inflorescentia mascula angusta, petalis $3-4 \mathrm{~mm}$ longis, receptaculo masculo longiore quam latiore c. 3 mm longo differt. - Typus: Kadir, North Bor. For. Dept. A2520 (holo K; iso BO, KEP).

Male inflorescences light brown pubescent; raceme slender, unbranched, many (1040)-flowered; bracts lanceolate, 3-6 mm long; pedicels 3-8 $\mathrm{mm}$ long, hardly longer than bracts. Male perianth 5-6 mm diam.; receptacle tube c. 3 by c. $2 \mathrm{~mm}$, sepals c. $2 \mathrm{~mm}$ long, petals $3-4$ by $3 \mathrm{~mm}$; filaments (0.5-) $1 \mathrm{~mm}$ long, anthers (thecae) $1.5-2$ mm long.

Distribution - Sabah, known from 2 collections only (Sandakan and Tawau).

Habitat \& Ecology - Climber in peat swamp forest; lowlands.

Note - The inflorescences of Elmer 20472 is more lax than in the type, slightly approaching those of var. borneensis, but its flowers are similar to those in the type, Kadir A2520.

Specimens studied:

Elmer 20472, male, Oct. 1922-March 1923, Tawau (deviating by \pm lax inflorescence); Kadir A2520, male, flowers white, 06-02-1949, Sandakan, marsh (type).

\section{ACKNOWLEDGEMENTS}

We thank the Director of Sabah Parks allowing us to study Cucurbitaceae in the Kinabalu Park and vicinity, and in Tawau Hill Park. The Director of the Sepilok Herbarium generously provided transport and companions in the field. Herbarium material in BM, BO, K, KEP, L, Sabah Parks Herbarium Kinabalu and SAN was studied. Dr. J.F. Veldkamp and J.H. van Os (both Leiden) provided the Latin diagnosis and the drawings, respectively.

Fig. 2. a-h: Baijiania borneensis A.M. Lu \& J.Q. Li var. borneensis. a. Male flower; a'. male flower, opened, showing stamens; b, b', b". stamens: adaxial, abaxial and lateral view, respectively; c. twig with female inflorescence; d. detail of female inflorescence; e. female flower; e'. female flower, opened, showing style with 3-lobed stigma and staminodes; f. petal with basal staminodes; g. twig with infructescence; h. seed. - i-j": B. borneensis A.M. Lu \& J.Q. Li var. paludicola Duyfjes. i. Male flower; $i^{\prime}$. male flower, opened, showing stamens; $j, j^{\prime}, j^{\prime \prime}$. stamens: adaxial, abaxial and lateral view, respectively (a-b": SAN (De Wilde \& Duyfjes) 141929; c-h: SAN (De Wilde \& Duyfjes) 141932; i-j": Kadir A2520; all drawn by Jan van Os). 


\section{REFERENCES}

Beaman, J.H., C. Anderson \& R.S. Beaman. 2001. The plants of Mount Kinabalu 4. Dicotyledon families Acanthaceae to Lythraceae: 206-213, pl. 15 (B, C) - 17. Natural History Publications, Kota Kinabalu.

Jeffrey, C. 1962. Notes on Cucurbitaceae, including a proposed new classification of the family. Kew Bull. 15: 337-371.

Li, J.Q. 1993. A revision of the genus Siraitia Merr. and two new genera of Cucurbitaceae. Acta Phytotax. Sin. 31: 45-55.

Lu, A.M. \& Zhi Y. Zhang. 1984. The genus Siraitia Merr. in China. Guihaia 4: 27-33.

Merrill, E.D. 1929. Plantae Elmerianae Borneenses. Cucurbitaceae. Univ. Calif. Publ. Bot. 15: 298-300.

Zhang, Zhi Y. \& A.M. Lu. 1989. Pollen morphology of the subtribe Thladianthinae (Cucurbitaceae) and its taxonomic significance. Cathaya 1: 23-35.

\section{INDEX TO SPECIMENS}

$1=$ Baijiania borneensis (Merr.) A.M. Lu \& J.Q. Li var. borneensis

2 = Baijiania borneensis (Merr.) A.M. Lu \& J.Q. Li var. paludicola Duyfjes

Clemens 26749: 1.

Elmer 20472: 2.

Kadir A2520: 2 (type) - KEP (Taleon): 55285: 1 - Kuswata 835: 1.

Pereira et al. JTP 470: 1.

RSNB (Chew et al.) 242: 1.

SAN (Madani \& Kodoh) 81462: 1; (Madani \& Saigol) 90200: 1; (Sumbing \& Matin) 103547: 1; (Sumbing) 120278: 1; (De Wilde \& Duyfjes) 139471: 1; 141917: 1; 141921: 1; 141928: 1; 141929: 1; 141932: $1 ; 144004: 1$.

Villamil 308: 1 (type). 